\title{
Rising Rate of Liver Transplantation in the Baby Boomer Generation with Non-alcoholic Steatohepatitis in the United States
}

\author{
Osama Siddique ${ }^{1}$, Mairin Joseph-Talreja ${ }^{2}$, Eric R. Yoo ${ }^{3}$, Ryan B. Perumpail ${ }^{2}$, \\ George Cholankeril ${ }^{4}$, Stephen A. Harrison ${ }^{5}$, Zobair M. Younossi ${ }^{6}$, Robert J. Wong ${ }^{7}$ \\ and Aijaz Ahmed*2
}

\begin{abstract}
${ }^{1}$ Department of Medicine, Memorial Hospital of Rhode Island/Alpert Medical School of Brown University, Providence, RI, USA; ${ }^{2}$ Division of Gastroenterology and Hepatology, Stanford University School of Medicine, Stanford, CA, USA; ${ }^{3}$ Department of Medicine, University of Illinois College of Medicine, Chicago, IL, USA; ${ }^{4}$ Division of Gastroenterology and Hepatology, University of Tennessee Health Sciences Center, Memphis, TN, USA; ${ }^{5}$ Radcliffe Department of Medicine, University of Oxford, Oxford, United Kingdom; ${ }^{6}$ Department of Medicine, Center for Liver Diseases, Inova Fairfax Hospital, Falls Church, VA, USA; ${ }^{7}$ Division of Gastroenterology and Hepatology, Alameda Health System, Highland Hospital, Oakland, CA, USA
\end{abstract}

\section{Abstract}

Background and Aims: Nonalcoholic steatohepatitis (NASH) is the most rapidly growing indication for liver transplantation (LT) in the United States and is on a trajectory to become the leading indication for $L T$ in the next decade. We aimed to study the trends in NASH-related LT among persons born between 1945 and 1965, the baby boomer (BB) generation. Methods: We performed a retrospective cohort analysis using population-based data from the United Network for Organ Sharing/Organ Procurement and Transplantation Network registry from 2004-2015 to evaluate the birth cohort-specific trends in liver transplant waitlist registrations and liver transplant surgeries in patients with NASH. We stratified our study population into three birth cohorts: 1) birth before 1945, 2) birth between 1945 and 1965, and 3) birth after 1965. Results: The overall rates of NASHrelated waitlist registrations and liver transplant surgeries steadily increased from 2004 to 2015 and were reflective of a sharp rise noted in the NASH BB sub-group. From 2004 to 2015, the proportion of BB patients with NASH added to LT waitlist demonstrated an incremental growth, $60.6 \%$ in 2004 versus $83.2 \%$ in $2015(p<0.01)$. Among the liver transplant recipients with $\mathrm{NASH}$, the proportion represented by the BB cohort increased from $56.3 \%$ in 2004 to $80.0 \%$ in $2015(p<0.01)$. Conclusion: We report rising rates of waitlist registration and LT for the indication of NASH. More importantly, the BB sub-cohort was mainly responsible for these alarming trends.

Keywords: Baby boomers; Liver transplantation; Non-alcoholic steatohepatitis; Waitlist.

Abbreviations: ALD, alcoholic cirrhosis; BB, baby boomer; BMI, body mass index; HCC, hepatocellular carcinoma; HCV, hepatitis C virus; LT, liver transplantation; MELD, Model for End-Stage Liver Disease; NAFLD, non-alcoholic fatty liver disease; NASH, nonalcoholic steatohepatitis; OPTN, Organ Procurement Transplant Network; UNOS, United Network for Organ Sharing; US, United States. Received: 10 January 2017; Revised: 26 April 2017; Accepted: 10 May 2017

*Correspondence to: Aijaz Ahmed, Division of Gastroenterology and Hepatology, Stanford University School of Medicine, 750 Welch Road, Suite \#210, Stanford, CA 94304, USA. Tel: +1-650-498-5691, Fax: +1-650-498-5692, E-mail: aijazahmed@stanford.edu
Citation of this article: Siddique $O$, Joseph-Talreja $M$, Yoo ER, Perumpail RB, Cholankeril G, Harrison SA, et al. Rising rate of liver transplantation in the baby boomer generation with nonalcoholic steatohepatitis in the United States. J Clin Transl Hepatol 2017;5(3):193-196. doi: 10.14218/JCTH.2017.00003.

\section{Introduction}

In the United States (US), non-alcoholic fatty liver disease (NAFLD) is estimated to be the most common cause of chronic liver disease, affecting between 80 and 100 million individuals, among whom nearly $25 \%$ have non-alcoholic steatohepatitis (NASH). ${ }^{1}$ Although its etiology is unknown, NASH occurs in individuals who drink little or no alcohol and is typically associated with characteristic features of metabolic syndrome, such as central obesity, impaired glucose tolerance, high levels of triglycerides, and low levels of high-density lipoprotein. ${ }^{2}$ Histologically, NASH is characterized by progressive hepatocellular injury, demonstrating hepatic lipid deposition, lobular inflammation, balloon degeneration, and fibrosis. NASH is a clinically progressive subset of NAFLD and may result in cirrhosis with risk of developing into hepatocellular carcinoma (HCC) and end-stage liver disease necessitating liver transplantation (LT).$^{3-5}$ Fortunately, simple steatosis, the more prevalent component of NAFLD, is non-progressive, but its clinical relevance is not welldefined. ${ }^{1}$

Due to the rise in obesity rates in the US and globally over the last two decades, the prevalence of NASH is expected to increase. NASH is currently the most rapidly growing indication for LT in the US and predicted to become the most common etiology for LT in the near future. ${ }^{5-7} \mathrm{~A}$ recent study which utilized data from the United Network for Organ Sharing (UNOS)/Organ Procurement Transplant Network (OPTN) showed that in 2013, NASH became the second leading indication among adults awaiting LT in the US. ${ }^{6}$ The aging of the US baby boomer (BB) generation-birth cohort 1945-1965-in the backdrop of the obesity epidemic poses increasing challenges in defining the optimal clinical management of this special population with $\mathrm{NASH}$. The trends in 
$\mathrm{NASH}$-related waitlist registrations for LT and liver transplant surgeries among the BB sub-cohort in the US remain unknown.

\section{Methods}

In our retrospective analysis, we evaluated adult patients (age 18 years or older) who were waitlisted for liver transplant surgery or underwent LT in the US from 2004-2015. Patients with acute liver failure, simultaneous listing for multiple organs, and re-transplantation were excluded. The etiology of chronic liver disease leading to liver transplant waitlist registration and surgery were determined based on diagnostic coding in the UNOS/OPTN database. NASH was added as a diagnostic code in the UNOS/OPTN database in 2001. Patients with NASH were estimated based on previously published criteria. ${ }^{6,7}$

To determine the trends and frequency of waitlist registrations and surgeries in NASH-related LT among the BB birth cohort, we stratified our study population into three birth cohorts: 1) birth before 1945, 2) birth between 1945 and 1965, and 3) birth after 1965. We analyzed the trends and frequency of liver transplant waitlist registrations and liver transplant surgeries in these three sub-cohorts. The study time period was selected to restrict the analysis to Model for End-Stage Liver Disease (MELD) score era.

Approval from the Institutional Review Board was not needed at our tertiary care center and academic institution for analyzing data from de-identified national registry. Categorical variables were tested using chi-square tests. Continuous variables were tested using Student's t-test. All missing data were omitted. Statistical significance was met with a two-tailed $p$-value of $<0.05$. All statistical analyses were performed using the Stata statistical package (version 10; Stata Corp. LLC, College Station, TX, US).
Siddique O. et al: NASH liver transplantation in baby boomers

\section{Results}

Patient characteristics, demographics and other clinically relevant variables were compared and were found to be evenly distributed across the three birth sub-cohorts. From 2004 to 2014, the proportion of BB with NASH added to LT waitlist demonstrated an incremental growth as shown in Fig. 1 (60.6\% in 2004, $70.6 \%$ in 2009, $p<0.05$; and $84.3 \%$ in $2014, p<0.05)$. Correspondingly, a decrease in proportion of listings in the post-BB cohort (those born after 1965) (36.4\% in 2004, 23.2\% in 2009, $p<0.05$; and $5.6 \%$ in 2014, $p<0.001$ ) as well as a steady uptrend in pre-BB cohort (those born before 1945) $(3.0 \%$ in $2004,6.2 \%$ in 2009, $p=0.754$; and $10.1 \%$ in 2014, $p=0.568$ ) was observed. Among liver transplant recipients with $\mathrm{NASH}$, similar trends were observed with the proportion represented by the BB cohort rising from $63.5 \%$ in 2004 to $65.9 \%$ in 2009 , $p=0.491$; and $82.9 \%$ in $2014, p<0.001$ (Fig. 1). Overall annual trends in NASH-related waitlist registrations and LT showed a steady growth from 2004 to 2014. These trends were reflective of a dominant influence of NASH BB subcohort.

We performed a sub-analysis on post-transplant outcomes on all patients undergoing LT during the study period and noted that $32.9 \%$ had body mass index (BMI) of $\geq 30 \mathrm{~kg} / \mathrm{m}^{2}$. While patients in all obesity classes had similar survival to patients with BMI $18.0-24.9 \mathrm{~kg} / \mathrm{m}^{2}$, the presence of concurrent diabetes mellitus resulted in significantly lower posttransplant survival. Patients with NASH were noted to have a higher prevalence of diabetes compared to other common etiologies of chronic liver disease.

We also performed a sub-analysis using OPTN/UNOS waitlist registration data from 2004 and 2013 to provide a perspective by comparing absolute numbers on an annual basis in patients with NASH. Furthermore, trends in annual waitlist registrations for NASH were compared with alcoholic cirrhosis (ALD) and hepatitis C virus (HCV) infection. Our subanalysis showed that the waitlist registrants with $\mathrm{NASH}$ increased $170 \%$ (from 804 to 2174 ), while ALD increased

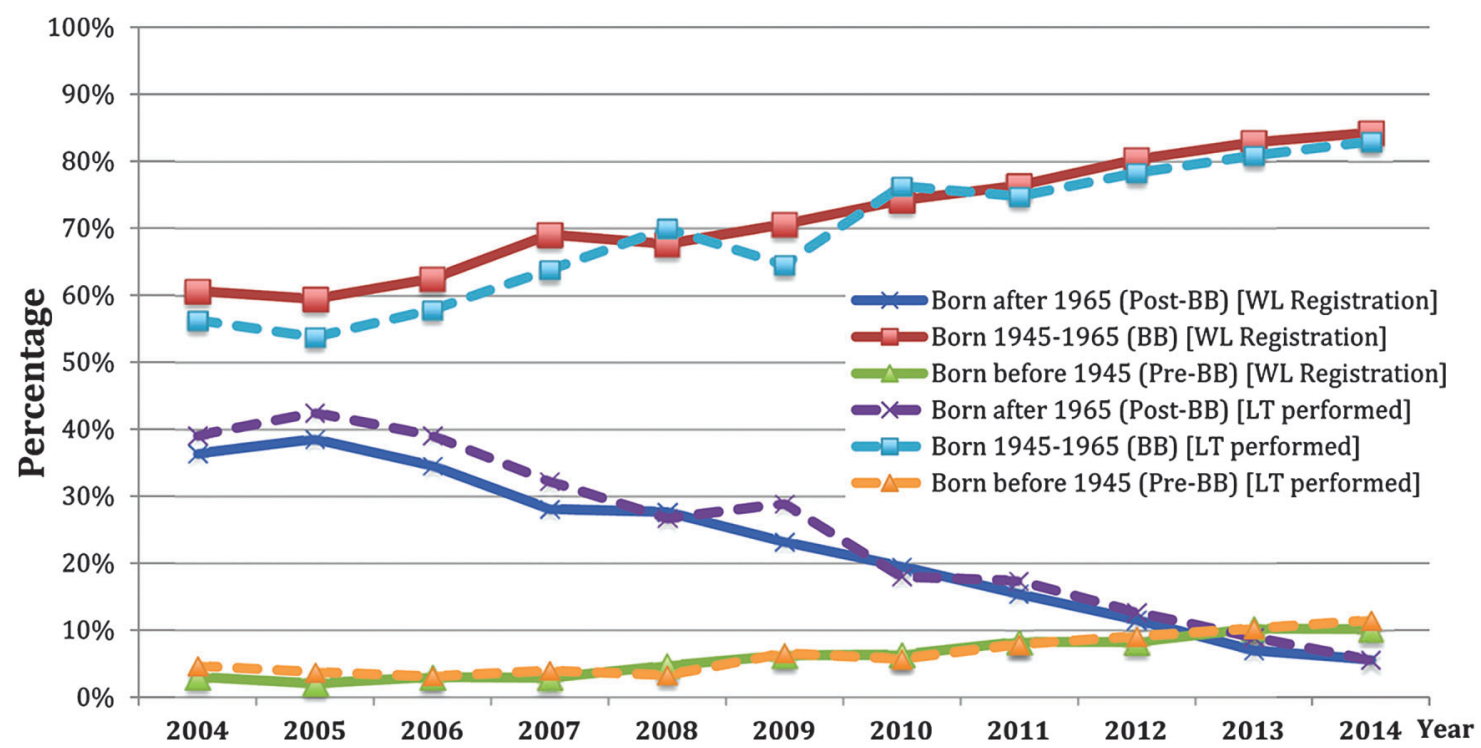

Fig. 1. Annual waitlist registrations for non-alcoholic steatohepatitis (NASH) and annual NASH-related liver transplants by birth cohorts from $2004-2014$. 
$45 \%$ (from 1400 to 2024), and HCV infection increased only $14 \%$ (from 2887 to 3291). In 2013, NASH was the secondleading disease indication among liver transplant waitlist registrants, with $\mathrm{HCV}$ infection as the leading indication. The highest proportion of LT waitlist registrations for HCV-related liver disease was noted in BB cohort mimicking NASH trends noted above. Based on multivariate analysis, patients with ALD were less likely to die within 90 days when compared to patients with $\mathrm{NASH}$ (odds ratio $=0.77 ; 95 \%$ confidence interval: $0.67-0.89 ; p<0.001)$; and, patients with $\mathrm{HCV}$ infection had similar odds for 90-day waitlist survival compared with NASH patients.

\section{Discussion}

NASH-related waitlist registrations and LT continue to grow, with the BB cohort demonstrating an incremental increase in the rate for waitlist registrations and liver transplant surgeries. Due to the asymptomatic nature of most patients with NASH and lack of cost-effective screening protocols, identification of NASH sub-cohorts at risk for progression to endstage liver disease requiring LT is warranted. Further studies are needed with a longitudinal design to identify risk factors in patients with NASH associated with progression to end-stage liver disease and necessitating LT. Given the strong association of NASH with metabolic syndrome and the growing obesity epidemic, NASH is increasingly being recognized as a major contributor to morbidity and mortality in the US. ${ }^{8}$ Progressive liver damage due to NASH is more marked in patients with underlying insulin resistance and/or diabetes mellitus. ${ }^{9}$ With NASH expected to become the leading etiology for LT in the US, targeted screening and intervention is necessary in at-risk sub-populations. Our study identified BB birth cohort as a sub-population at a high risk for NASHrelated hepatic complications.

Globally, the prevalence of NAFLD has exceeded $25 \% .{ }^{10,11}$ However, the reliance on current BMI cut-offs for defining obesity and subsequent risk of NASH has significant limitations. Several population-based studies have shown significant rates of NAFLD and/or NASH in non-obese patients with BMI $<30 \mathrm{~kg} / \mathrm{m}^{2} .{ }^{12}$ Prevalence studies in the non-transplant NASH population may not be applicable to patients awaiting LT with ascites as a confounding factor. Ascites contributes to BMI and is not corrected for in the UNOS/OPTN dataset. It is important to note that greater than $80 \%$ of new waitlist registrants for LT have ascites. ${ }^{6}$ A large, prospective, multicenter study showed that ascites lead to frequent misclassification of BMI, with up to $10 \%$ of patients with Class I obesity (BMI $30-35 \mathrm{~kg} / \mathrm{m}^{2}$ ) requiring correction into a lower BMI classification. ${ }^{13}$ This may also lead to misclassification of other etiologies of chronic liver diseases such as NASH. Furthermore, duration and severity of malnutrition (hypoalbuminemia and muscle wasting) in the setting of end-stage liver disease may lower the BMI in patients with NASH.

The inherent limitations of registry-based data must be discussed. First and foremost, the assignment of primary and secondary diagnoses of chronic liver disease among LT recipients is based entirely on UNOS-coded data, and is not necessarily subject to valid confirmatory measures. As a result, errors in miscoding or errors in diagnosis entry may occur. However, as commented in previous studies utilizing the UNOS/OPTN registry data, any errors in diagnostic coding that have occurred are more likely to be non-differential in nature, favoring the bias in our analysis towards the null hypothesis. Our study is limited by its cross-sectional design. NASH as a diagnostic category was added to the UNOS database in 2001. In addition, greater awareness and recognition of NASH as a significant burden of chronic liver disease may have also led to more accurate diagnosis of $\mathrm{NASH}$, and thereby may have contributed to some degree of selection bias during the study period. While our study utilized BMI $\geq 30 \mathrm{~kg} / \mathrm{m}^{2}$ among patients with cryptogenic cirrhosis and unknown etiology to define our NASH category, it may underestimate the true prevalence of $\mathrm{NASH}$. For example, there are racial/ethnic variations in populations at risk of NASH associated with increasing BMI, especially among Asians where overweight and obesity may be more accurately categorized at lower cut-offs compared to other ethnicities and may have further contributed to underestimating the true prevalence of NASH diagnoses. Furthermore, the presence of ascites may further complicate the true estimation of BMI among patients with cirrhosis. Finally, data on hyperlipidemia, other cardiovascular risk factors, and medication intake are not collected in the UNOS/OPTN database and remain a limitation of our analysis.

\section{Conclusions}

In summary, the increasing obesity epidemic has contributed to the rising prevalence of NASH and associated hepatic complications in the US. While HCV still remains the leading indication for LT in the US, NASH is the most rapidly growing indication for LT. Clinical implications of our observations are significant and will present the medical community with a management challenge of liver transplant evaluation in an aging population with increasing prevalence of diabetes. Although NASH and alcoholic liver disease had a noticeably similar number of LT since 2008, NASH remains the second leading indication for LT. Targeted commitment and resources are needed to halt the detrimental impact of NASH on at-risk sub-populations. We need to bolster our efforts to raise community awareness and institute primary prevention measures through support groups, educate healthcare providers at all levels to improve the earlier recognition and diagnosis of NAFLD, and alert stakeholders and policy makers to pay attention to the growing epidemic of obesity and related complications, such as $\mathrm{NASH}$-related liver failure.

\section{Conflict of interest}

The authors have no conflict of interests related to this publication.

\section{Author contributions}

Study concept and design (OS, MJT, ERY, RBP, GC, SAH, ZMY, RJW, AA), acquisition of data (OS, MJT, ERY, RBP, GC, AA), analysis and interpretation of data (OS, MJT, ERY, RBP, GC, SAH, ZMY, RJW, AA), drafting of the initial and final manuscript (OS, MJT, ERY, RBP, GC, AA), critical revision of the manuscript (OS, MJT, ERY, RBP, GC, SAH, ZMY, RJW, AA), and study supervision (AA).

\section{References}

[1] Ahmed A, Wong RJ, Harrison SA. Nonalcoholic fatty liver disease review: diagnosis, treatment, and outcomes. Clin Gastroenterol Hepatol 2015;13: 2062-2070. doi: 10.1016/j.cgh.2015.07.029. 
[2] DeFilippis AP, Blaha MJ, Martin SS, Reed RM, Jones SR, Nasir K, et al. Nonalcoholic fatty liver disease and serum lipoproteins: the Multi-Ethnic Study of Atherosclerosis. Atherosclerosis 2013;227:429-436. doi: 10.1016/j. atherosclerosis.2013.01.022.

[3] Chalasani N, Younossi Z, Lavine JE, Diehl AM, Brunt EM, Cusi K, et al. The diagnosis and management of non-alcoholic fatty liver disease: practice guideline by the American Gastroenterological Association, American Association for the Study of Liver Diseases, and American College of Gastroenterology. Gastroenterology 2012;142:1592-1609. doi: 10.1053/j.gastro.2012. 04.001.

[4] White DL, Kanwal F, El-Serag HB. Association between nonalcoholic fatty liver disease and risk for hepatocellular cancer, based on systematic review. Clin Gastroenterol Hepatol 2012;10:1342-1359.e2. doi: 10.1016/j.cgh.2012. 10.001

[5] Charlton MR, Burns JM, Pedersen RA, Watt KD, Heimbach JK, Dierkhising RA. Frequency and outcomes of liver transplantation for nonalcoholic steatohepatitis in the United States. Gastroenterology 2011;141:1249-1253. doi: 10.1053/j.gastro.2011.06.061.

[6] Wong RJ, Aguilar M, Cheung R, Perumpail RB, Harrison SA, Younossi ZM, et al. Nonalcoholic steatohepatitis is the second leading etiology of liver disease among adults awaiting liver transplantation in the United States. Gastroenterology 2015;148:547-555. doi: 10.1053/j.gastro.2014.11.039.

[7] Wong R], Cheung R, Ahmed A. Nonalcoholic steatohepatitis is the most rapidly growing indication for liver transplantation in patients with hepato- cellular carcinoma in the U.S. Hepatology 2014;59:2188-2195. doi: 10. 1002/hep. 26986.

[8] Vernon G, Baranova A, Younossi ZM. Systematic review: the epidemiology and natural history of non-alcoholic fatty liver disease and non-alcoholic steatohepatitis in adults. Aliment Pharmacol Ther 2011;34:274-285. doi: $10.1111 / j .1365-2036.2011 .04724 . x$.

[9] Ahmed A, Perumpail RB, Harrison SA. High prevalence of hepatic fibrosis in the setting of coexisting diabetes and hepatic steatosis: A case for selective screening in the general population? Hepatology 2016;63:20-22. doi: 10 . 1002/hep.28277.

[10] Loomba R, Sanyal AJ. The global NAFLD epidemic. Nat Rev Gastroenterol Hepatol 2013;10:686-690. doi: 10.1038/nrgastro.2013.171.

[11] Younossi ZM, Koenig AB, Abdelatif D, Fazel Y, Henry L, Wymer M. Global epidemiology of nonalcoholic fatty liver disease-Meta-analytic assessment of prevalence, incidence, and outcomes. Hepatology 2016;64:73-84. doi: 10.1002/hep.28431.

[12] Das K, Das K, Mukherjee PS, Ghosh A, Ghosh S, Mridha AR, et al. Nonobese population in a developing country has a high prevalence of nonalcoholic fatty liver and significant liver disease. Hepatology 2010;51:1593-1602. doi: 10.1002/hep.23567.

[13] Leonard J, Heimbach JK, Malinchoc M, Watt K, Charlton M. The impact of obesity on long-term outcomes in liver transplant recipients-results of the NIDDK liver transplant database. Am J Transplant 2008;8:667-672. doi: 10. 1111/j.1600-6143.2007.02100.x. 